\title{
The Requirement for Enhanced CREB1 Expression in Consolidation of Long-Term Synaptic Facilitation and Long-Term Excitability in Sensory Neurons of Aplysia
}

\author{
Rong-Yu Liu, Leonard J. Cleary, and John H. Byrne \\ Department of Neurobiology and Anatomy, W. M. Keck Center for the Neurobiology of Learning and Memory, The University of Texas Medical School at \\ Houston, Texas 77030
}

Accumulating evidence suggests that the transcriptional activator cAMP response element-binding protein 1 (CREB1) is important for serotonin (5-HT)-induced long-term facilitation (LTF) of the sensorimotor synapse in Aplysia. Moreover, creb1 is among the genes activated by CREB1, suggesting a role for this protein beyond the induction phase of LTF. The time course of the requirement for CREB1 synthesis in the consolidation of long-term facilitation was examined using RNA interference techniques in sensorimotor cocultures. Injection of CREB1 small-interfering RNA (siRNA) immediately or $10 \mathrm{~h}$ after 5-HT treatment blocked LTF when measured at 24 and $48 \mathrm{~h}$ after treatment. In contrast, CREB1 siRNA did not block LTF when injected $16 \mathrm{~h}$ after 5-HT treatment. These results demonstrate that creb1 expression must be sustained for a relatively long time to support the consolidation of LTF. In addition, LTF is also accompanied by a long-term increase in the excitability (LTE) of sensory neurons (SNs). Because LTE was observed in the isolated SN after 5-HT treatment, this long-term change was intrinsic to that element of the circuit. LTE was blocked when CREB1 siRNA was injected into isolated SNs immediately after 5-HT treatment. These data suggest that 5-HTinduced CREB1 synthesis is required for consolidation of both LTF and LTE.

\section{Introduction}

The induction of long-term memory (LTM) and the process of consolidating a new memory require the activation of transcription factors that lead to expression of genes necessary for synaptic plasticity and the growth of new synaptic connections (Alberini, 2009). The cellular and molecular mechanisms underlying LTM have been extensively studied in monosynaptic connections between identified sensory neurons (SNs) and motor neurons (MNs) that mediate withdrawal reflexes of Aplysia (Zhao et al., 2003; Sharma and Carew, 2004; Lee et al., 2007; Fioravante et al., 2008; Kandel, 2009). Application of serotonin (5-HT), a neurotransmitter that mimics sensitization training, facilitates this synaptic connection (Montarolo et al., 1986; Emptage and Carew, 1993; Zhang et al., 1997). Accumulating evidence suggests that the cAMP response element-binding protein (CREB) family of transcription factors serves a key role in regulating gene expression required for long-term facilitation (LTF) and LTM (Dash et al., 1990; Bartsch et al., 1998; Liu et al., 2008; Alberini, 2009). In Aplysia, the transcription factor CREB1 is phosphory-

\footnotetext{
Received Sept. 24, 2010; revised Feb. 21, 2011; accepted March 17, 2011.

Author contributions: R.-Y.L., L.J.C., and J.H.B. designed research; R.-Y.L. performed research; R.-Y.L. and L.J.C. analyzed data; R.-Y.L., L.J.C., and J.H.B. wrote the paper.

This research was supported by National Institutes of Health Grant NS019895. We thank J. Liu and E. Kartikaningrum for preparing the cultures, A. Tarakad, J.-Y. Park, and D. Axelson for assistance with the experiments, A. Netek and P. Smolen for their comments on this manuscript, L. Morales for help with the illustrations, and B. Kaang for providing plasmids.

Correspondence should be addressed to Dr. John H. Byrne, Department of Neurobiology and Anatomy, W. M. Keck Center for the Neurobiology of Learning and Memory, The University of Texas Medical School at Houston, Houston, TX 77030. E-mail: John.H.Byrne@uth.tmc.edu.

DOI:10.1523/JNEUROSCI.5071-10.2011

Copyright $\odot 2011$ the authors $\quad 0270-6474 / 11 / 316871-09 \$ 15.00 / 0$
}

lated and activated subsequent to 5-HT application, and activation of gene expression by CREB1 is essential for LTF (Bartsch et al., 1998; Liu et al., 2008).

creb1 expression is itself activated by $5-\mathrm{HT}$, and elevated levels are observed up to $24 \mathrm{~h}$ after treatment (Bartsch et al., 1998; Mohamed et al., 2005; Liu et al., 2008). CREB1 binds to the promoter of its own gene, suggesting that positive feedback, mediated by activated CREB1, contributes to the sustained activation of this gene (Mohamed et al., 2005). Moreover, blocking this putative feedback by injection of CREB1 antibody into the presynaptic SNs after 5-HT treatment blocked LTF (Liu et al., 2008). Therefore, it appears that a persistent increase in CREB1 levels, sustained by this positive-feedback loop, helps to maintain gene activation essential for the consolidation of LTF. However, it is not known for how long CREB1 synthesis is critically involved in the consolidation process. The main goal of the present study is to examine the temporal window during which the enhanced synthesis of CREB1 is required for LTF.

In addition to effects on synaptic strength, sensitization training and 5-HT also produce an increase in the intrinsic excitability of SNs (Dale et al., 1987; Cleary et al., 1998), which is an independent mechanism for memory storage (for review, see Mozzachiodi and Byrne, 2010). A persistent question is whether all of the effects of training in SNs are mediated by the same intracellular pathways. Therefore, we also examined the potential contribution of CREB1 to the increase in long-term excitation (LTE).

Both of these questions were examined by injecting CREB1 small-interfering RNA (siRNA) into the SNs, in sensorimotor ( $\mathrm{SN}-\mathrm{MN})$ cocultures and in isolated SNs, at different time points after 5-HT treatment. RNA interference (RNAi) blocked both 
LTF and LTE, suggesting a common requirement for the 5-HTinduced expression of CREB1.

\section{Materials and Methods}

CREB1 siRNA. The CREB1a sequence (Bartsch et al., 1998) was used by Dharmacon Research to design and synthesize a pool of CREB1 siRNAs (containing four different sequences to ensure mRNA target knockdown). The pooled CREB1 siRNA are designed to target the following sequences in creb1: ${ }^{279}$ GAGCUGUCCUCUCCAGUAU ${ }^{297},{ }^{306} U G A U U-$ CAAACAGUAGUCAA ${ }^{324},{ }^{325} A G U C A A G A C G G C C A C G A U U^{343}$, and ${ }^{417}$ CUAACAAUGACAAACGCUA ${ }^{435}$. Nontargeting siRNA obtained from the same company was used as injection control, which is referred to in the manuscript and illustrations as "control siRNA" or "consiRNA." siRNAs were resuspended into siRNA buffer provided by Dharmacon Research.

Cell culture, 5-HT treatment, and siRNA injections. Isolated SNs or $\mathrm{SN}-\mathrm{MN}$ cocultures were prepared according to conventional procedures (Schacher and Proshansky, 1983; Chin et al., 2002). SNs were isolated from the ventrocaudal cluster of the pleural ganglion. MNs used in coculture were isolated from the abdominal ganglion of juvenile animals. Dishes of SN cultures were plated with 5-10 SNs. Dishes of SN-MN cocultures were plated with a single $\mathrm{SN}$ and a single MN. Both $\mathrm{SN}$ cultures and SN-MN cocultures were allowed to grow for $5 \mathrm{~d}$ at $18^{\circ} \mathrm{C}$, and the growth medium was replaced before treatments and recordings with a solution of $50 \% \mathrm{~L} 15$ and $50 \%$ artificial seawater (ASW) (in mм: 450 $\mathrm{NaCl}, 10 \mathrm{KCl}, 11 \mathrm{CaCl}_{2}, 29 \mathrm{MgCl}_{2}$, and 10 HEPES, pH 7.6). LTF was induced by $5-\mathrm{HT}$ treatment as described previously (Liu et al., 2008). Five 5-min pulses of either vehicle (L15/ASW) or $50 \mu \mathrm{M} 5$-HT (Sigma) were applied to the bath with an interstimulus interval of $20 \mathrm{~min}$. Isolated SNs were used for immunofluorescence and excitability experiments to examine the effects of 5-HT on a defined cell type. The presence of an MN in the cocultures would not allow us to determine whether the effects of 5 - $\mathrm{HT}$ on the $\mathrm{SN}$ were attributable to direct actions on the $\mathrm{SN}$ or to indirect actions through the MN.

For injection, siRNA ( $5 \mu \mathrm{M}$ final concentration) was dissolved in a solution containing $100 \mathrm{~mm} \mathrm{KCl}$ and $2.5 \mathrm{mg} / \mathrm{ml} 70 \mathrm{kDa}$ fluoresceindextran (Invitrogen). The solution was injected into the cytoplasm of SNs using an Eppendorf InjectMan NI 2 coupled to a FemtoJet microinjection system. Injection efficiency and locus were monitored with a Carl Zeiss Axiovert 100TV fluorescence microscope. Injections were deemed successful if the cytoplasm and processes from SNs were filled with fluorescein (injection dye). Cultures were excluded from additional use if fluorescein appeared in the nucleus.

All treatments and siRNA injections were performed blind, as were measurements of fluorescence intensity.

CRE-EGFP reporter. A CRE-enhanced green fluorescent protein (EGFP) reporter vector was prepared as described previously (Liu et al., 2008). Cultured SNs were treated with either 5-HT or vehicle as described above. Eighteen hours after the end of the treatment, the CREEGFP expression vector $(1 \mu \mathrm{g} / \mu \mathrm{l})$ was injected into the nucleus. The injection buffer contained $2.5 \mathrm{mg} / \mathrm{ml} 70 \mathrm{kDa}$ Texas Red-dextran to monitor the efficiency and locus of injection. Six hours after vector injection ( $24 \mathrm{~h}$ after the end of the treatment), cells were fixed with $4 \%$ paraformaldehyde in PBS containing 30\% sucrose and processed for microscopic analysis as described below. EGFP signal in the cell body and red fluorescence from injection dye in the nucleus were determined by tracing the outline of the cell body and nucleus, respectively. Mean fluorescence intensity for EGFP was normalized to the amount of plasmid injected, indicated by the intensity of injected fluorescent marker in the nucleus. Measurements from the 5-10 viable cells in each dish were averaged. The number of samples in each experiment $(n)$ reported in Results indicates the number of dishes. The normalized EGFP expression in 5-HT-treated cells was compared with the normalized EGFP expression in vehicle-treated cells. Data were analyzed with a paired Student's $t$ test using SigmaStat software (Jandel Scientific).

Immunofluorescence and confocal microscopy for CREB1. For experiments that examined levels of protein, cells were grown on coverslips in culture dishes, were fixed at various time points after 5-HT or vehicle treatment, and processed for confocal microscopy. Briefly, cells were fixed in a solution of 4\% paraformaldehyde in PBS containing 30\% sucrose. Cells expressing EGFP (see above) were rinsed and passed on directly for confocal microscopy. For immunofluorescence, fixed cells were rinsed in PBS and blocked for $30 \mathrm{~min}$ at room temperature in Superblock buffer (Pierce)/0.2\% Triton X-100/3\% normal goat serum and subsequently incubated overnight at $4^{\circ} \mathrm{C}$ with primary antibody. Two antibodies were used. One was directed against all forms of CREB1 (tCREB1), and the other was directed specifically against the phosphorylated form (pCREB1) (Mohamed et al., 2005). Antibodies were diluted 1:500 in blocking solution. Secondary antibody (1:200 dilution, goat anti-rabbit IgG conjugated to cyanine 3; Jackson ImmunoResearch) was applied in the same blocking solution for $1 \mathrm{~h}$ at room temperature. All cells processed for confocal microscopy were mounted with Prolong antifade medium (Invitrogen). Images were obtained with a Carl Zeiss LSM 510 confocal microscope using a $63 \times$ oil-immersion lens. A $z$-series of optical sections through the cell body ( $0.5 \mu \mathrm{m}$ increments) was taken, and the section through the middle of the nucleus was used for analysis of mean fluorescence intensity with MetaMorph Offline software (Universal Imaging Corporation). Five to 10 neurons on each dish were analyzed, and measurements from neurons in the same dish were averaged. The number of samples $(n)$ reported in Results indicates the number of dishes. For statistical analysis, intensity of immunoreactivity for tCREB1 or pCREB1 in 5-HT-treated dishes was compared with that in vehicletreated dishes, and data were analyzed with a paired Student's $t$ test.

Electrophysiology. Stimulation of presynaptic SNs was performed extracellularly using a blunt patch electrode filled with L15/ASW. Intracellular recordings from MNs were made with 10-20 M $\Omega$ sharp electrodes filled with $3 \mathrm{~m}$ potassium acetate connected to an Axoclamp 2-B amplifier (Molecular Devices) (Angers et al., 2002). Data acquisition and analyses of resting potential, input resistance, and EPSP amplitude were performed with pClamp 8 software (Molecular Devices). MNs were current clamped at $-90 \mathrm{mV}$ before measurement of EPSPs. Pretreatment measurements of EPSP amplitudes varied from 5 to $40 \mathrm{mV}$. Cultures were excluded from additional use if EPSPs were $<5 \mathrm{mV}$ or sufficiently large to trigger an action potential. MNs that had resting potentials more positive than $-30 \mathrm{mV}$ or input resistances $<10 \mathrm{M} \Omega$ were also excluded from the analyses. Immediately and at various times (10 and 16-18 h) after the end of treatment with vehicle or 5-HT, siRNA was injected into the SN. Cultures were returned to culture medium after injection. At 24 and $48 \mathrm{~h}$ after treatment with 5 -HT or vehicle, resting potential, input resistance of the MN, and EPSP amplitude were assessed in the same manner as the baseline measurements. In cases in which the posttest EPSP amplitude was accompanied by an action potential, the EPSP amplitude was assigned a value of $45 \mathrm{mV}$ because the largest synaptic potential obtained in our culture system never exceeded that amplitude. For statistical analysis, the amplitudes of the EPSPs at 24 and $48 \mathrm{~h}$ after treatment (posttest) were normalized to the EPSPs measured before treatment (pretest). Data were analyzed by ANOVA followed by post hoc analysis with Student-Newman-Keuls tests using SigmaStat software (Jandel Scientific).

Excitability of SNs was measured in a separate group of experiments using isolated SNs. Neurons were impaled with a single microelectrode (10-20 M $\Omega$ resistance) and current clamped at $-45 \mathrm{mV}$. Input resistance was measured by applying $0.1 \mathrm{nA}$ of hyperpolarizing current for $2 \mathrm{~s}$. Firing threshold was measured by applying $1 \mathrm{~s}$ of depolarizing current in increasing increments of $0.1 \mathrm{nA}$ until an action potential was triggered. The lowest current intensity necessary to fire a single action potential was considered the firing threshold. Excitability was measured by counting the number of action potentials triggered by applying $0.5 \mathrm{nA}$ of depolarizing current for $1 \mathrm{~s}$. When the firing threshold of SNs was $\geq 0.5 \mathrm{nA}$ but $<1.0 \mathrm{nA}, 1.0 \mathrm{nA}$ of depolarizing current was used to measure excitability. When the firing threshold was $\geq 1.0 \mathrm{nA}$ but $<2.0 \mathrm{nA}, 2.0 \mathrm{nA}$ of depolarizing current was used. SNs were excluded from additional use if cells had resting potentials more positive than $-30 \mathrm{mV}$ or failed to respond to depolarizing current up to $2.0 \mathrm{nA}$. The number of samples $(n)$ reported in Results indicate numbers of cells recorded. Treatment protocols and data analysis were similar to those described above for analysis of EPSPs.

All treatments and siRNA injections were performed blind, as were measurements of EPSPs and other electrophysiological properties. 
A1 tCREB1

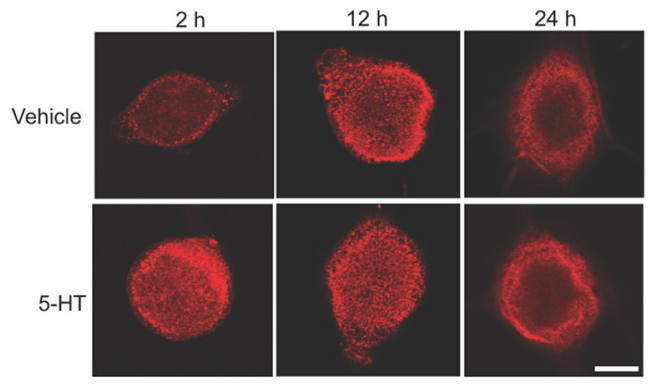

A2

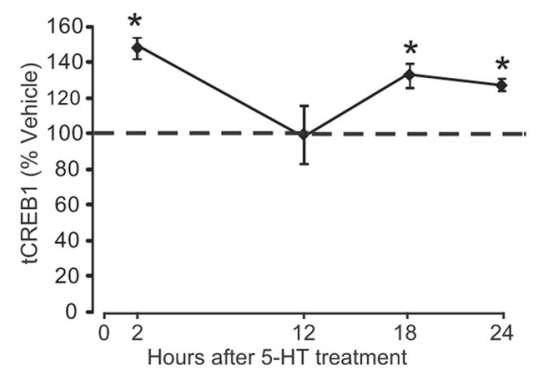

B1 CRE-mediated gene expression

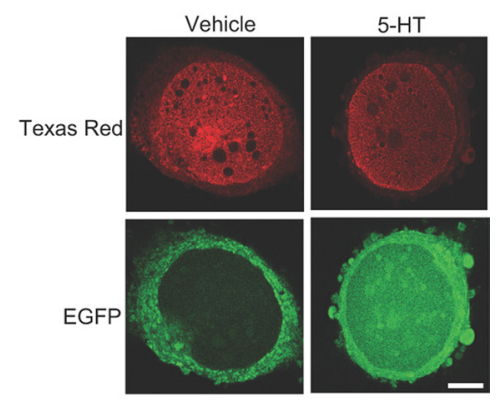

B2

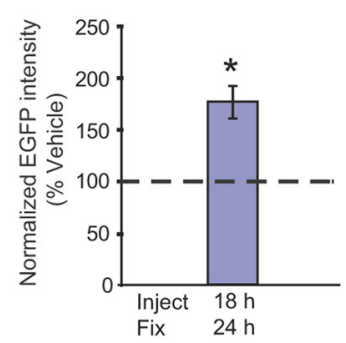

Figure 1. Serotonin-induced changes in tCREB1 and CRE-mediated gene expression. $A, 5-H T$ treatment induced tCREB1 expression for at least $24 \mathrm{~h}$ in cultured SNs. $\boldsymbol{A 1}$, Representative confocal images of tCREB1 immunofluorescence in SNs at 2, 12, and $24 \mathrm{~h}$ after treatment with vehicle or $5-\mathrm{HT}$. Scale bar, $30 \mu \mathrm{m}$. $\mathbf{A 2}$, Summary data. CREB1 was elevated at $2 \mathrm{~h}$, returned to basal level at $12 \mathrm{~h}$, and increased again at 18 and $24 \mathrm{~h}$ after 5 -HT treatment $\left({ }^{*} p<0.05\right)$. B. (RE-mediated EGFP expression was enhanced $18-24$ $\mathrm{h}$ after 5-HT treatment. B1, Representative images of sensory cells injected with CRE-EGFP reporter vector $18 \mathrm{~h}$ after 5-HT or vehicle treatment and fixed $6 \mathrm{~h}$ later. Texas Red- dextran was added to the injection buffer to confirm nuclear injection. Scale bar, $15 \mu \mathrm{m}$. B2, 5-HT-induced EGFP expression was calculated as the ratio of the 5-HT-treated to vehicle-treated samples for each experiment. EGFP expression was significantly increased in neurons after 5 -HT treatment compared with vehicle-treated cells $\left({ }^{*} p<0.05\right)$, demonstrating that CRE-dependent gene expression is still enhanced $18 \mathrm{~h}$ after 5 -HT treatment.

\section{Results}

Dynamic changes of total CREB1 protein in sensory neurons after 5-HT treatment

CREB1 mRNA and protein levels in pleural-pedal ganglia are increased for at least $24 \mathrm{~h}$ after induction of LTF (Liu et al., 2008). To determine whether the observed increase of CREB1 in the ganglia is specifically present in the subpopulation of neurons critical for LTM, we examined the levels of CREB1 protein in isolated SNs using immunofluorescence and confocal microscopy. Previous studies showed that total CREB1 protein levels were increased in the cell body (including cytoplasm and nucleus) $2 \mathrm{~h}$ after the end of treatment with 5-HT (Liu et al., 2008). To further examine the dynamics of CREB1 levels, immunofluorescence was performed at longer time periods after treatment. The 5-HT-induced changes in CREB1 were compared with controls that were exposed to vehicle and fixed at the same time points. Using the same treatment protocol, we found that CREB1 levels increased in two phases. At $2 \mathrm{~h}$ after exposure to 5-HT, CREB1 levels in the cell body increased to $150 \pm 6 \%$ (mean \pm SEM) of time-matched control (Fig. $1 A)\left(n=5, t_{(4)}=4.60, p<\right.$ $0.05)$. However, CREB1 levels returned to baseline at $12 \mathrm{~h}$ after 5-HT (Fig. 1A) $\left(99.5 \pm 16 \%, n=5, t_{(4)}=0.96, p>0.05\right)$. A second phase of increase in CREB1 levels was observed at $18 \mathrm{~h}$ $\left(132 \pm 7 \%, n=4, t_{(3)}=5.45, p<0.05\right)$ and persisted through at least $24 \mathrm{~h}$ after treatment $\left(128 \pm 3 \%, n=6, t_{(5)}=6.65, p<0.01\right)$. Although we focused on the amount of CREB1 in the entire cell body, changes in the level of nuclear CREB1 followed the same trends. Nuclear staining of CREB1 was significantly increased at $2 \mathrm{~h}\left(153 \pm 19 \%\right.$ of control, $\left.t_{(4)}=4.366, p<0.05\right)$, returned to baseline at $12 \mathrm{~h}\left(99 \pm 15 \%, n=5, t_{(4)}=0.96, p>0.05\right)$, and significantly increased again at $18 \mathrm{~h}\left(134 \pm 9 \%, n=4, t_{(3)}=\right.$
4.124, $p<0.05)$ and $24 \mathrm{~h}$ after 5-HT treatment $\left(130 \pm 5 \%, n=6, t_{(5)}=\right.$ 3.284, $p<0.05)$.

It is noteworthy that the CREB1 level is increased at $24 \mathrm{~h}$ after 5-HT treatment, supporting the hypothesis that the late expression of CREB1 may be important for maintaining the synthesis of new proteins essential for consolidation of LTF and LTE.

\section{CREB1 mediated gene expression: CRE-EGFP expression at 18-24 h after 5-HT}

We previously reported that the increase in CREB1 is regulated by CREB1 itself, corroborating the existence of a CREB1positive feedback loop. Moreover, this loop drives CRE-dependent gene transcription for at least $12-17 \mathrm{~h}$ after LTF induction (Liu et al., 2008). Elevations in CREB1 protein levels in isolated SNs were detected at 18 and $24 \mathrm{~h}$ after 5-HT treatment (Fig. 1A2). Can increases in CREB1 at this late stage of LTF still mediate CREdependent gene transcription? Because CREB1 must be activated by phosphorylation (Lonze and Ginty, 2002), we first examined the 5-HT-induced phosphorylation of Ser-85 (equivalent to Ser-133 in vertebrate CREB) at $18 \mathrm{~h}$ after 5 -HT treatment, using an antibody directed against phosphorylated CREB1 (Mohamed et al., 2005). Levels of phospho-CREB1 immunoreactivity in the cytoplasm and processes were extremely low compared with nuclear levels, indicating that CREB1 phosphorylation is primarily a nuclear event. Compared with vehicle control, nuclear pCREB1 levels were increased by $21 \pm 7 \%\left(n=4, t_{(3)}=5.05, p<0.05\right)$ at $18 \mathrm{~h}$ after 5 -HT, suggesting that phosphorylation of CREB1 is enhanced for a prolonged period after treatment. This finding is consistent with the hypothesis that regulation of gene expression by CREB1 is required for the consolidation phase of LTF and LTE.

The increase in the level of phosphorylation of CREB1 at $18 \mathrm{~h}$ after 5-HT may lead to increased CRE-mediated transcription. To directly test this possibility, we injected a CRE-EGFP reporter vector at $18 \mathrm{~h}$ after 5 -HT treatment into isolated SNs. Six hours later (corresponding to $24 \mathrm{~h}$ after the end of the treatment), cells were fixed and processed for confocal imaging. We found that EGFP expression was increased to $176 \pm 29 \%\left(n=6, t_{(5)}=2.64\right.$, $p<0.05$ ) of that in vehicle-treated cells (Fig. $1 B$ ). Together, these results indicate that CRE-mediated transcription is not limited to the induction phase of LTF, and CRE-mediated protein synthesis persists for at least $18-24 \mathrm{~h}$ after application of 5-HT.

\section{CREB1 siRNA injection after 5-HT blocked 5-HT-induced synthesis of CREB1}

Previous results suggest that 5-HT-induced synthesis of CREB1 is necessary for LTF (Liu et al., 2008). In those experiments, LTF was blocked by injection of an antibody against total CREB1 immediately after 5-HT treatment. However, one limitation of that technique is the possibility that the antibody also interferes with the function of preexisting basal levels of CREB1. To more specifically assess the function of newly synthesized CREB1, we 
A 1 tCREB1 immunostaining $2 \mathrm{~h}$ after 5-HT treatment and after siRNA injection

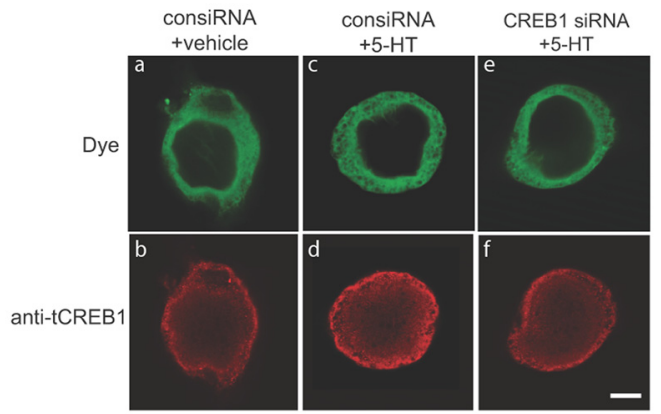

A2

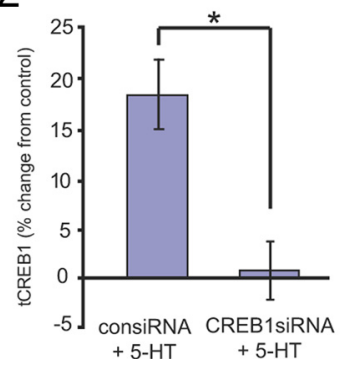

B Basal tCREB1 level 48 and $72 \mathrm{~h}$ after CREB1 siRNA injection

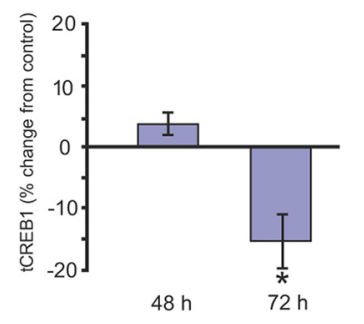

Figure 2. CREB1 siRNA injection blocked 5-HT-induced increase in tCREB1 expression without affecting basal levels. A1, Confocal images of neurons injected with CREB1 siRNA or control siRNA. siRNAs were injected immediately after vehicle or 5 -HT treatment. Fluorescein-dextran was coinjected with siRNAs to monitor cytoplasmic injection $(\boldsymbol{a}, \boldsymbol{c}, \boldsymbol{e})$. At $2 \mathrm{~h}$ after the end of treatment, cells were fixed and processed for immunostaining for CREB1 $(\boldsymbol{b}, \boldsymbol{d}, \boldsymbol{f})$. Scale bar, 20 $\mu \mathrm{m}$. A2, Data were expressed as the ratios of tCREB1 levels in 5-HT-treated cells to tCREB1 levels in vehicle-treated control siRNA cells. Summary data indicated that the 5 -HT-induced increase in CREB1 can be blocked by CREB1 siRNA ( $\left.{ }^{*} p<0.05\right)$. $\boldsymbol{B}$, CREB1 siRNA or control siRNA was injected into untreated SNs. Forty-eight or $72 \mathrm{~h}$ later, cells were processed for tCREB1 immunofluorescence. Summary data show that injection of CREB1 siRNA did not affect the basal level of tCREB1 $48 \mathrm{~h}$ later but did significantly decrease the basal level $72 \mathrm{~h}$ later compared with the time-matched control siRNA-injected groups ( $\left.{ }^{*} p<0.05\right)$.

used RNAi to inhibit induction of new CREB1 synthesis. Either CREB1 siRNA or control siRNA was injected into the cytoplasm of isolated SNs immediately after treatment with 5-HT. A third group of cells were injected with control siRNA immediately after treatment with vehicle. Two hours later, cells were fixed and levels of CREB1 were assessed using immunofluorescence. The ratio of 5-HT-induced tCREB1 levels in CREB1 siRNAinjected cells to vehicle-treated cells was calculated, as was the ratio of 5-HT-induced tCREB1 level in control siRNAinjected cells to vehicle-treated cells. Cells treated with 5-HT and injected with control siRNA showed a $18 \pm 3 \%$ increase in CREB1 in the cell bodies, whereas CREB1 levels in cells treated with 5 -HT but injected with CREB1 siRNA were $1 \pm 3 \%$ of vehicle-treated cells in the cell bodies (Fig. $2 A$ ). The difference between the levels of CREB1 in these two groups of cells was statistically significant $\left(n=4\right.$; cell body staining, $t_{(3)}=3.29$, $p<0.05)$. Changes in the level of nuclear CREB1 followed the same trends. Compared with control siRNA-injected and vehicle-treated cells, cells treated with 5 -HT and injected with control siRNA showed $24 \pm 6 \%$ increase in CREB1 in the nucleus, whereas nuclear CREB1 levels in cells treated with 5 -HT but injected with CREB 1 siRNA were $5 \pm 3 \%$ of vehicletreated cells. The difference between the levels of nuclear CREB1 in these two groups of cells was statistically significant $\left(n=4, t_{(3)}=6.934, p<0.01\right)$. Therefore, CREB1 siRNA blocked the increase in CREB1 measured in both cell body and nucleus at $2 \mathrm{~h}$ after treatment, a rapid effect.
We also monitored the basal level of CREB1 protein at 48 and $72 \mathrm{~h}$ after CREB1 siRNA injection in untreated SNs. At $48 \mathrm{~h}$ after injection, the basal level of CREB1 in CREB1 siRNA-injected cells was not statistically different from that of control siRNA-injected cells (Fig. $2 B)(4 \pm 2 \%$ change in tCREB1 compared with control siRNA-injection, $\left.n=5, t_{(4)}=2.307, p>0.05\right)$. However, at $72 \mathrm{~h}$ after injection, CREB1siRNA significantly reduced the basal level of CREB1 by $15 \pm 4 \%\left(n=6, t_{(5)}=3.26, p<0.05\right)$. Thus, CREB1 siRNA injected immediately after 5-HT treatment does not affect the basal level of CREB1 for a relatively long period of time (at least $48 \mathrm{~h}$ ), suggesting that any effects of CREB1 siRNA during this time are attributable to inhibition of newly synthesized protein and that the protein has a relatively long half-life. Eventually (i.e., by $72 \mathrm{~h}$ ), normal protein turnover reduces the basal level.

\section{Blocking synthesis of CREB1 immediately after 5-HT treatment blocked LTF}

Having determined that CREB1 siRNA was effective in blocking the 5-HT-induced increase in CREB1 protein levels, we examined the functional significance of both the first and second phases of CREB1 synthesis by injecting CREB1 siRNA immediately after the initial induction of LTF. Briefly, basal synaptic strength (pretest) was recorded in $\mathrm{SN}-\mathrm{MN}$ cocultures. The cocultures were then treated with five pulses of vehicle or 5-HT, using the same application protocol that increased CREB1 expression. Immediately after the end of treatment, CREB1 or control siRNA was pressure injected into the cytoplasm of the SN (Fig. 3A). The amplitude of the EPSP was measured 24 and $48 \mathrm{~h}$ after treatment (posttest) (Fig. $3 B$ ).

Two-way ANOVA indicated a significant interaction between treatments and siRNA injections at $24 \mathrm{~h}$ after treatment (Fig. $3 \mathrm{C}$ ) (percentage \pm SEM change in EPSP: control siRNA + vehicle, $-20 \pm 9 \%, n=6$; control siRNA + 5-HT, $102 \pm 46 \%, n=7$; CREB1 siRNA + vehicle, $7 \pm 8 \%, n=7$; CREB1 siRNA + 5-HT, $9 \pm 16 \%, n=6 ; F_{(1,22)}=4.66$ for interaction, $p=0.042 ; F_{(1,22)}=$ 1.67 for siRNA injections, $p=0.21 ; F_{(1,22)}=5.67$ for treatments, $p=0.029)$. Subsequent pairwise comparisons using StudentNewman-Keuls post hoc tests indicated that 5-HT induced facilitation in the control siRNA-injected cocultures (5-HT + control siRNA vs vehicle + control siRNA, $q=4.5, p<0.05)$. In addition, there was a significant difference between the amplitude of the EPSPs in the 5-HT-treated control siRNA-injected cells and amplitude of EPSPs in the 5-HT-treated CREB siRNA-injected cells $(q=3.45, p<0.05)$, suggesting that the effects of 5-HT were blocked by CREB1 siRNA injection. Moreover, there was no difference between the vehicle-treated control siRNA-injected cells and the vehicle-treated CREB1 siRNA-injected cells $(q=0.86$, $p>0.05$ ), indicating that injection of CREB1 siRNA alone did not significantly affect synaptic transmission over a $24 \mathrm{~h}$ period. Finally, there were no significant differences in basal synaptic strength (pretest) among the four groups (one-way ANOVA, $\left.F_{(3,25)}=0.78, p>0.05\right)$. In addition, the passive properties of the motor neurons were not significantly different among the groups (resting potentials, $F_{(3,25)}=2.077, p>0.05$; input resistances, $\left.F_{(3,25)}=2.13, p>0.05\right)$ at $24 \mathrm{~h}$ after treatment. Therefore, the observed differences in facilitation between CREB1 siRNAinjected and control siRNA-injected neurons were not attributable to differences in basal synaptic strength or to biophysical properties of the MN. These results suggest that disruption of new CREB1 synthesis impaired LTF at $24 \mathrm{~h}$.

A significant interaction was also observed between treatments and siRNA injections at $48 \mathrm{~h}$ after treatment (Fig. 3D) (control siRNA + vehicle, $-15 \pm 16 \%, n=7$; control siRNA + 
A

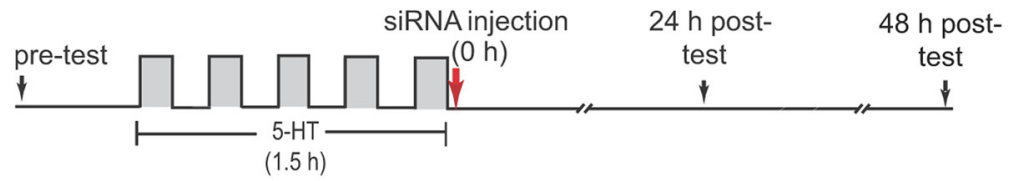

B
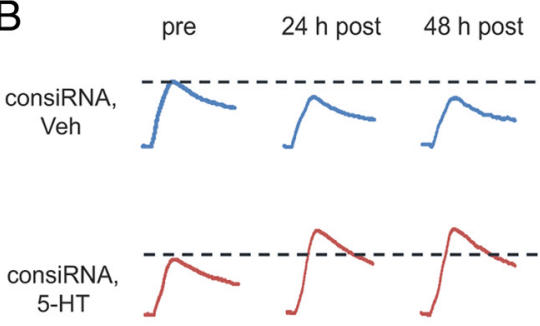

C $24 \mathrm{~h}$

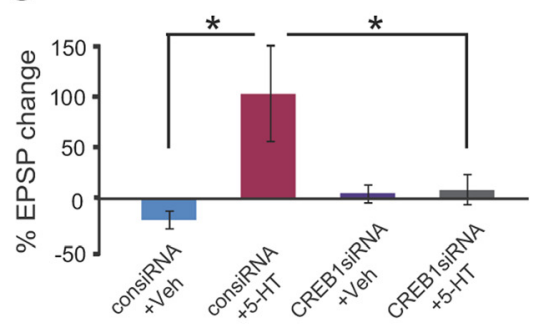

D $48 \mathrm{~h}$
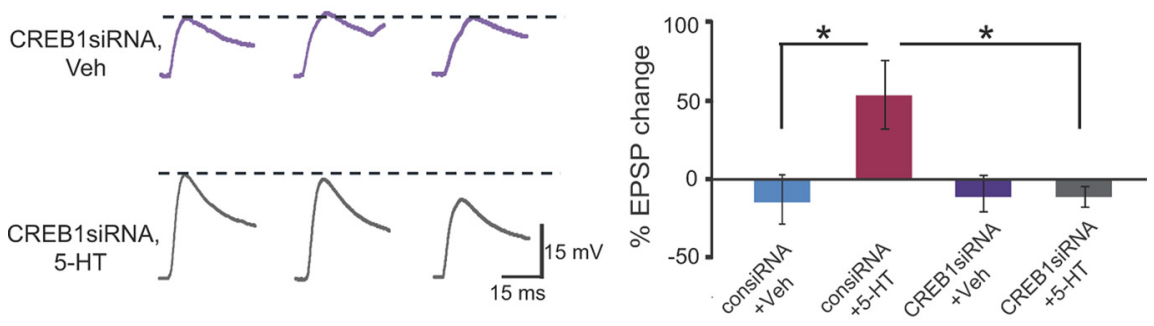

Figure 3. CREB1 siRNA injection immediately after 5-HT treatment blocked LTF. $\boldsymbol{A}$, Protocol for CREB1 or control siRNA injection and electrophysiological testing. siRNAs were injected into the cytosol of SNs immediately after the end of treatment with 5-HT or vehicle. $\boldsymbol{B}$, Representative traces of EPSPs before (pre) and 24 and $48 \mathrm{~h}$ after treatment with 5-HT or vehicle (Veh). C, D, Summary data. Two-way ANOVA followed by post hoc tests indicated that injection of CREB siRNA blocked LTF measured at $24(\boldsymbol{C})$ and $48 \mathrm{~h}(\boldsymbol{D})$ without significantly affecting basal synaptic transmission $\left({ }^{*} p<0.05\right)$.

$5-\mathrm{HT}, 62 \pm 22 \%, n=6$; CREB1 siRNA + vehicle, $-11 \pm 12 \%$, $n=6$; CREB 1 siRNA +5 -HT, $-11 \pm 7 \%, n=6 ; F_{(1,21)}=5.04$ for interaction, $p=0.036 ; F_{(1,21)}=4.02$ for siRNA injections, $p=0.06$; $F_{(1,21)}=5.02$ for treatments, $\left.p=0.036\right)$. Subsequent pairwise comparisons indicated that 5 -HT induced significant facilitation in the control siRNA-injected cocultures $(5-\mathrm{HT}+$ control siRNA vs vehicle + control siRNA, $q=4.57, p<0.05)$. In addition, there was a significant difference in the amplitude of EPSPs elicited by 5 -HT-treated control siRNA-injected cells and the amplitude of EPSPs elicited by 5 -HT-treated CREB1 siRNA-injected cells $(q=4.2, p<0.05)$. These results suggest that disruption of the CREB1 feedback loop immediately after 5-HT impaired LTF at $48 \mathrm{~h}$. In addition, the passive properties of the motor neurons were not significantly different among the groups (resting potentials, $F_{(3,24)}=0.516, p>0.05$; input resistances, $\left.F_{(3,24)}=0.33, p>0.05\right)$ at $48 \mathrm{~h}$ after treatment. Therefore, blocking synthesis of CREB1 shortly after the induction of LTF impaired consolidation of LTF when tested either 24 or 48 h later without affecting basal synaptic transmission or passive membrane properties.

\section{Time window during which LTF is sensitive to CREB1 siRNA injection}

To explore the contribution of the second phase of CREB1 elevation to consolidation of LTF at 24 and $48 \mathrm{~h}$, we injected CREB1 siRNA $10 \mathrm{~h}$ after 5-HT treatment. This time point was chosen to ensure that the siRNA would be active at a time before the start of the second phase of increased CREB1 levels (Fig. 1A2). We first examined the effects of CREB1 siRNA injection on 5-HTinduced increases in CREB1 protein $24 \mathrm{~h}$ after 5-HT treatment in isolated SNs. Either CREB1 siRNA or control siRNA was injected into the cytoplasm of SNs $10 \mathrm{~h}$ after treatment with 5-HT. A third group of cells was injected with control siRNA $10 \mathrm{~h}$ after treatment with vehicle. Fourteen hours later (i.e., $24 \mathrm{~h}$ after the end of treatment), cells were fixed and levels of CREB1 were assessed by immunofluorescence. The CREB1 levels in 5-HT-treated groups were normalized to those in the control siRNA-injected and vehicle-treated group. Cells treated with 5-HT and injected with consiRNA showed a $25 \pm 5 \%$ increase in CREB1 in the cell bodies, whereas CREB1 levels in cells treated with 5-HT but injected with CREB1 siRNA showed a $-4 \pm 5 \%$ change in CREB1 (Fig. 4A). The changes in nuclear CREB1 were similar to the changes in the whole cell body (percentage change of consiRNA + vehicle: consiRNA + 5-HT, $35 \pm 10 \%$; CREB1 siRNA + 5-HT, $-2 \pm$ $8 \%)$. These differences between the 5-HTinduced changes in CREB1 in both cell body and nucleus in these two groups of cells were statistically significant $(n=5$; cell body staining, $t_{(4)}=7.79, p<0.05$; nuclear staining, $\left.t_{(4)}=7.19, p<0.05\right)$. These results indicate that the second phase of CREB1 elevation is attributable to new protein synthesis.

We next examined the effects on LTF of injecting CREB1 siRNA $10 \mathrm{~h}$ after 5-HT treatment (Fig. $4 \mathrm{~B}$ ). Because injection of CREB1 siRNA itself immediately after treatment with vehicle did not alter basal synaptic strength up to $48 \mathrm{~h}$ after 5-HT (Fig. 3D), the CREB1 siRNA + vehicle treatment group was not included in these electrophysiological experiments. We observed a significant difference in the degree of facilitation among the three groups at $24 \mathrm{~h}$ after treatment (Fig. 4C) (percentage \pm SEM change: control siRNA + vehicle, $-1 \pm 7 \%$, $n=6$; control siRNA +5 -HT, $80 \pm 20 \%, n=9$; CREB1 siRNA + 5 -HT, $-2 \pm 16 \%, n=8$; one-way ANOVA, $F_{(2,22)}=8.19, p<$ $0.05)$. Subsequent pairwise comparisons indicated a significant difference between the amplitude of EPSPs in the 5-HT-treated control siRNA-injected cells and the amplitude of EPSPs in the 5 -HT-treated CREB1 siRNA-injected cells $(q=5.1, p<0.05)$, suggesting that 5 -HT-induced LTF was blocked by CREB1 siRNA injection. As expected, compared with vehicle control, 5-HT induced significant facilitation in the control siRNA-injected cocultures $(q=4.60, p<0.05)$. Importantly, there were no significant differences in basal synaptic strength (pretest) among the three groups (one-way ANOVA, $F_{(2,22)}=0.89, p>$ $0.05)$. In addition, the passive properties of the motor neurons were not significantly different among the groups (resting potentials, $F_{(2,22)}=3.18, p>0.05$; input resistances, $F_{(2,22)}=$ $0.74, p>0.05)$ at $24 \mathrm{~h}$ after treatment. Therefore, the observed differences in facilitation between CREB1 siRNA-injected and control siRNA-injected neurons were not attributable to differences in basal synaptic strength or changes in the two biophysical properties of the MNs.

We also observed a significant difference among the three groups at $48 \mathrm{~h}$ after treatment (Fig. $4 D$ ) (percentage \pm SEM change: control siRNA + vehicle, $-5 \pm 8 \%, n=7$; control siRNA + 5-HT, $51 \pm 16 \%, n=8$; CREB1 siRNA + 5-HT: $-1 \pm$ $9 \%, n=7$; one-way ANOVA, $\left.F_{(2,20)}=6.46, p<0.01\right)$. In contrast, the passive properties of the motor neurons were not 
significantly different among the groups (resting potentials, $F_{(2,20)}=0.53, p>$ 0.05 ; input resistances, $F_{(2,20)}=0.323, p>$ $0.05)$ at $48 \mathrm{~h}$ after treatment.

Subsequent pairwise comparisons of EPSP amplitudes indicated that, at $48 \mathrm{~h}$ after treatment, 5-HT induced significant facilitation in the control siRNA-injected cocultures $(q=4.38, p<0.05)$. Moreover, there was a significant difference between the amplitude of EPSPs in the 5-HT-treated control siRNA-injected cells and the amplitude of EPSPs in the 5-HTtreated CREB1 siRNA-injected cells $(q=$ $4.3, p<0.05)$. These results indicate that the second phase of CREB1 expression is essential for LTF.

Protein synthesis inhibitors, such as anisomycin, have a limited time window during which they are able to block synaptic plasticity and memory processing $(\mathrm{Da}-$ vis and Squire, 1984; Montarolo et al., 1986; Bekinschtein et al., 2007; Artinian et al., 2008). Therefore, we looked for a similar time window during which CREB1 is required for the consolidation of LTF. We injected CREB1 siRNA 16-18 $\mathrm{h}$ after 5-HT treatment (Fig. 5A) to block the subsequent synthesis of CREB1 (Fig. 1A2). Synaptic strength was assessed at 24 and $48 \mathrm{~h}$ after treatment with 5-HT or vehicle. We observed differences in the degree of facilitation among the three groups at $24 \mathrm{~h}$ (Fig. $5 B, C$ ) (percentage \pm SEM change: control siRNA + vehicle, $-6 \pm 9 \%, n=6$; control siRNA + 5-HT, $76 \pm$ $29 \%, n=9$; CREB1 siRNA +5 -HT, $37 \pm 8 \%, n=8$; one-way ANOVA, $\left.F_{(2,24)}=6.41, p<0.01\right)$. Subsequent pairwise comparisons indicated that 5-HT induced significant facilitation in the control siRNA-injected cocultures $(q=5.04, p<0.05)$. However, the 5-HT-induced facilitation in the control siRNA-injected cells was not significantly different from that of the CREB1 siRNAinjected cells $(q=2.46, p>0.05)$. Similarly, at $48 \mathrm{~h}$ after treatment, we observed differences in the degree of facilitation among the three groups (Fig. $5 B, D$ ) (percentage \pm SEM change: control siRNA + vehicle, $-8 \pm 13 \%, n=9$; control siRNA + 5-HT, $42 \pm 12 \%, n=7$; CREB siRNA + 5-HT, $33 \pm 12 \%, n=9$; one-way ANOVA, $F_{(2,22)}=$ $5.01, p<0.05)$. In contrast, the passive properties of the motor neurons were not significantly different among the groups (resting potentials, $F_{(2,22)}=1.169, p>0.05$; input resistances, $F_{(2,22)}=0.164$, $p>0.05)$ at $48 \mathrm{~h}$ after treatment.

Subsequent pairwise comparisons of the EPSP measurements indicated that 5-HT induced significant facilitation in the control siRNA-injected cocultures $(q=3.95, p<0.05)$. However, the 5 -HT-induced facilitation in the control siRNA-injected cells was not significantly different from that of the CREB1 siRNAinjected cells $(q=0.68, p>0.05)$. These results suggest that injection of CREB1 siRNA into the presynaptic SN at $16 \mathrm{~h}$ after 5-HT no longer induces a significant block of 5-HT-induced LTF measured at both 24 and $48 \mathrm{~h}$ after treatment. Thus, there appears to be a broad but discrete time window during which the increased synthesis of CREB1 is required for consolidation of LTF. The failure to block LTF also indicates that inhibition at earlier time points (Figs. 3, 4) was not attributable to "off-target" effects caused by unintended transcript silencing. Moreover, CREB1 siRNA injection itself has no significant effect on basal synaptic strength or SN excitability (see below), all sensitive measures of cell function and integrity.

\section{CREB1 siRNA injected immediately after 5-HT blocked long-term changes in cell excitability}

In addition to changes in synaptic strength, 5-HT also produces long-term changes in $\mathrm{SN}$ excitability. To test the hypothesis that CREB1 regulates both of these processes, we injected CREB1 siRNA into isolated SNs immediately after treatment with 5-HT or vehicle (Fig. 6A). Twenty-four and $48 \mathrm{~h}$ later, we measured several intrinsic cellular properties, including firing threshold, resting membrane, and input resistance. SN excitability was assessed by counting the number of spikes elicited by a constant-current pulse (Fig. $6 \mathrm{~B}$ ). A significant interaction between treatments and siRNA injections was found at $24 \mathrm{~h}$ after treatment (Fig. 6C) (percentage \pm SEM change: control siRNA + vehicle, $5 \pm 17 \%, n=17$; control siRNA +5 -HT, $170 \pm 55 \%$, $n=17$; CREB1 siRNA + vehicle, $-18 \pm 15 \%, n=25$; CREB1 siRNA + 5-HT, $21 \pm 24 \%, n=24$; two-way ANOVA, $F_{(1,76)}=$ 4.74 for interaction, $p=0.033 ; F_{(1,76)}=6.9$ for siRNA injections, $p=0.01 ; F_{(1,76)}=13.43$ for treatments, $\left.p<0.001\right)$. Subsequent pairwise comparisons revealed that, in control siRNA-injected neurons, 5-HT produced a significant increase in the number of spikes compared with vehicle treatment $(q=5.46, p<0.05)$ but not in CREB1 siRNA-injected neurons $(q=1.61, p>0.05)$. Moreover, the control siRNA +5 -HT group was significantly different from the CREB1 siRNA + 5-HT group $(q=4.72, p<$ $0.05)$, suggesting that inhibition of the CREB1 synthesis immediately after 5-HT impaired LTE at $24 \mathrm{~h}$. We also examined the changes in the firing threshold and did not find significant interaction between treatments and siRNA injections [mean \pm SEM change (in nA): control siRNA + vehicle, $0.05 \pm 0.05, n=16$; 
A

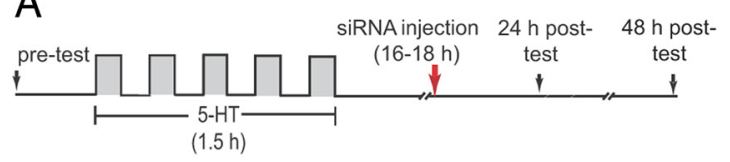

B
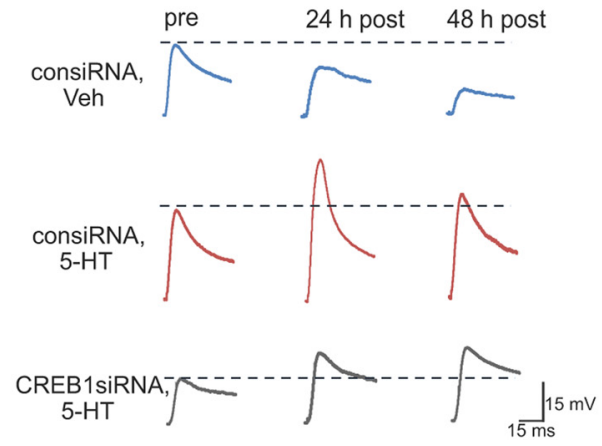

C $24 \mathrm{~h}$

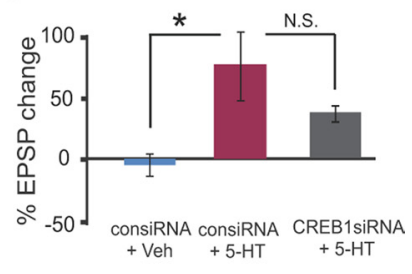

D $48 \mathrm{~h}$

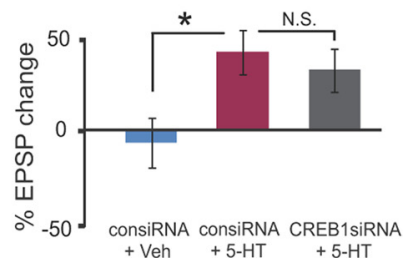

Figure 5. Injection of CREB1 siRNA $16 \mathrm{~h}$ after 5-HT treatment did not block LTF. A, Protocol for CREB1 or control siRNA injection and electrophysiological testing. siRNAs were injected into SNs in coculture at $16-18 \mathrm{~h}$ after the end of treatment with 5-HT or vehicle. $\boldsymbol{B}$, Representative traces of EPSPs recorded from cocultures before (pre) and 24 and $48 \mathrm{~h}$ after treatment with 5 -HT or vehicle (Veh). C, D, Summary data. One-way ANOVA followed by post hoc tests indicated that injection of CREB1 siRNA did not block LTF measured at $24 \mathrm{~h}(\boldsymbol{C})$ and $48 \mathrm{~h}(\boldsymbol{D})\left({ }^{*} p<0.05\right.$; N.S., not significant).

A
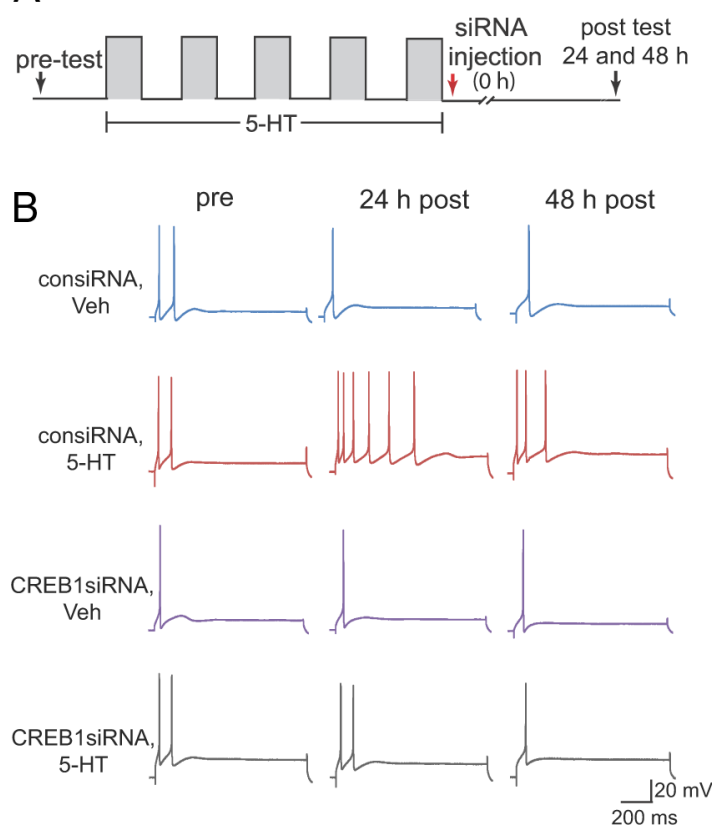
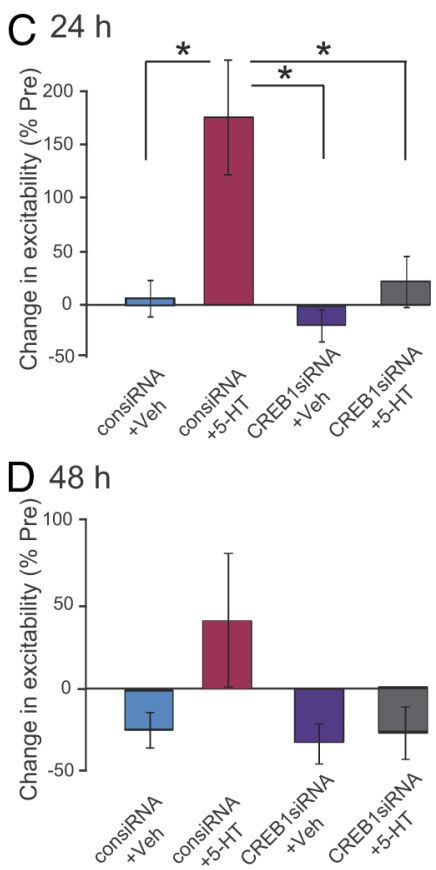

Figure 6. CREB1 siRNA blocked 5-HT-induced LTE in isolated SNs. $A$, Protocol for CREB1 or control siRNA injection and electrophysiological testing. siRNAs were injected into the cytosol of SNs immediately after the end of treatment with 5 -HT or vehicle. $B$, Action potentials were recorded from cultured SNs before (pre) and 24 and $48 \mathrm{~h}$ after treatment with 5 -HT or vehicle (Veh). C, Summary data ( $24 \mathrm{~h}$ after test). A significant increase in cell excitability was revealed in the 5 -HT-treated, control siRNA-injected group. The 5 -HT-induced changes in cell excitability were blocked by CREB1 siRNA injection ${ }^{*} p<0.05$ ). D, Summary data ( $48 \mathrm{~h}$ after test). The 5 -HT-induced increase in cell excitability was no longer detected.

control siRNA $+5-\mathrm{HT},-0.17 \pm 0.05, n=19 ; \mathrm{CREB} 1$ siRNA + vehicle, $0.14 \pm 0.06, n=25$; CREB1 siRNA +5 -HT, $0.04 \pm 0.05$, $n=22$; two-way ANOVA, $F_{(1,78)}=1.11$ for interaction, $p=$ $0.296 ; F_{(1,78)}=7.3$ for siRNA injections, $p=0.008 ; F_{(1,78)}=8.71$ for treatments, $p=0.004$ ]. Consistent with previous findings (Dale et al., 1987), 5-HT treatment did not cause significant changes in input resistance (one-way ANOVA, $F_{(3,81)}=0.458$, $p>0.05$ ) and resting membrane potential (one-way ANOVA, $\left.F_{(3,81)}=1.70, p>0.05\right)$.
We also examined SN excitability $48 \mathrm{~h}$ after 5-HT treatment (Fig. 6B,D). A trend toward increased excitability after 5-HT treatment was observed, but the changes were not statistically significant (two-way ANOVA, number of spikes: $F_{(1,81)}=2.16$ for interaction, $p=0.145 ; F_{(1,81)}=2.55$ for siRNA injections, $p=0.114 ; F_{(1,81)}=$ 2.45 for treatments, $p=0.122$ ) (Fig. $6 D$ ). Similarly, no changes in firing threshold were observed $48 \mathrm{~h}$ after treatment $\left(F_{(1,79)}=\right.$ 0.59 for interaction, $p=0.446 ; F_{(1,79)}=$ 2.08 for siRNA injections, $p=0.153$; $F_{(1,79)}=2.85$ for treatments, $\left.p=0.095\right)$. These results indicate that LTE induced by 5 -HT lasts $>24$ h but $<48$ h. Moreover, LTE can be induced in isolated SNs in the absence of MNs, indicating that postsynaptic factors are not required for the induction of LTE.

\section{Discussion}

In addition to its direct role in regulating synthesis of a number of proteins, CREB1 appears to regulate its own synthesis. The promoter region of the crebl gene contains a canonical CRE sequence, and 5-HT leads to increased binding of CREB1 to the promoter (Mohamed et al., 2005). Indeed, 5-HT produced a long-lasting enhancement of both mRNA and protein levels of CREB1 as well as its phosphorylation (Bartsch et al., 1998; Mohamed et al., 2005; Liu et al., 2008). These results are consistent with the idea that, after 5-HT treatment, CREB1 drives its own synthesis through a positive feedback loop (Mohamed et al., 2005). Support comes from experiments in which injection of antibody directed against CREB1 blocks LTF even when the injection occurs after 5-HT treatment (Liu et al., 2008).

In this paper, we provide additional evidence to support the hypothesis that CREB1 mediates LTF through a positive feedback loop. We demonstrated that expression of CREB1 protein is increased in cultured SNs, removing any doubt that CREB1 is modulated within the specific neurons that participate in the circuit exhibiting LTF. The increased levels of CREB1 are correlated with an increase in its active, phosphorylated form. Moreover, expression of a protein (EGFP) regulated by a CRE-dependent promoter was also enhanced by 5 -HT. In a previous paper, we blocked LTF by injecting anti-tCREB1 antibody into individual SNs (Liu et al., 2008). Although these results were consistent with the hypothesis that increased synthesis of CREB1 was important for LTF, we could not rule out the possibility that the antibody was blocking LTF by inhibiting the activity of preexisting CREB1 protein. Therefore, we used RNAi (Sharp, 2001; Milhavet et al., 2003) to disrupt the 5-HT-induced synthesis of CREB1 at specific times 
with the most specific effects. Our results demonstrate that both consolidation of LTF and LTE depend on new synthesis of CREB1.

\section{Dynamic changes of CREB1 after 5-HT treatment in isolated $\mathrm{SNs}$}

Previous experiments provided evidence that 5- $\mathrm{HT}$ regulates levels of CREB1, but those data were collected from heterogeneous populations of neurons (Bartsch et al., 1998; Mohamed et al., 2005). In this study, we examined pleural SNs, the specific sites of plasticity in the circuit. Our results were generally consistent with previous results, but we did observe an interesting difference between ganglia and isolated cells. In ganglia preparations, CREB1 protein levels decreased by $\sim 12 \mathrm{~h}$ after treatment but were still significantly greater than control and remained elevated for $24 \mathrm{~h}$ (Liu et al., 2008). In isolated cells, however, the CREB1 levels returned to baseline at $12 \mathrm{~h}$ and then increased again at 18 and $24 \mathrm{~h}$ (Fig. 1 A2). Therefore, there appear to be at least two distinct phases of CREB1 synthesis.

The second phase of CREB1 expression is functionally necessary for consolidation of LTF. Increased levels of tCREB1 were correlated with increased levels of pCREB1, the active form, $18 \mathrm{~h}$ after treatment. Increased tCREB1 levels were also correlated with enhanced expression of a CRE-dependent gene (Fig. 1B), suggesting that elevated CREB1 levels are driving enhanced expression. This is the first demonstration that CRE-mediated gene expression is enhanced $18 \mathrm{~h}$ after the induction of LTF.

The mechanism regulating the biphasic time course of CREB1 expression (Fig. 1 A2) is not currently known. CREB1 appears to be relatively stable in SNs, because CREB1 siRNA did not affect basal levels for at least $48 \mathrm{~h}$ (Fig. 2B). Therefore, we hypothesize that 5 -HT treatment also activates a process that degrades CREB1, driving levels back to baseline by $12 \mathrm{~h}$ after 5 -HT. This process may be similar to the degradation of the regulatory subunit of PKA mediated by ubiquitin (Hegde et al., 1993), the degradation of CCAAT/enhancer binding protein (ApC/EBP) (Yamamoto et al., 1999), and the degradation of the transcriptional repressor CREB1b (Upadhya et al., 2004). Presumably, the degradation is transient because expression is up again at 18 and $24 \mathrm{~h}$ after treatment. This new expression is associated with CREmediated gene expression (Fig. $1 B$ ) and is blocked by CREB1 siRNA (Figs. 2A, 4A).

\section{A limited time window for requirement of new CREB1 synthesis in the consolidation of LTF}

Injection of CREB1 siRNA immediately or $10 \mathrm{~h}$ after 5-HT treatment blocks enhancement of synaptic strength when measured at 24 and $48 \mathrm{~h}$ after treatment (Figs. 3, 4). CREB1 siRNA injected immediately after 5-HT treatment would interfere with new CREB1 synthesis in both phases, whereas CREB1 siRNA injected at $10 \mathrm{~h}$ after 5 -HT would only target CREB1 protein produced in the second phase. Therefore, it is clear that blocking the second phase impairs LTF. This finding raises an interesting question: what is the functional significance of CREB1 protein synthesized in the first phase? Clearly, the early expression of some proteins is essential for LTF. One example is ApC/EBP, an immediate early gene upregulated during the first $4 \mathrm{~h}$ after treatment with 5-HT (Alberini et al., 1994). Injection of a specific antibody to ApC/ EBP at 1, 6, and $9 \mathrm{~h}$, but not $12 \mathrm{~h}$, attenuates LTF (Alberini et al., 1994). Because ApC/EBP has a CRE in its promoter (Alberini et al., 1994), the early increase in the expression of CREB1 could help boost ApC/EBP expression. One way to resolve the early temporal requirement for $\mathrm{C} / \mathrm{EBP}$ and $\mathrm{CREB} 1$ and the late role for
CREB1 is to posit that the early expression of these and other genes, although necessary, is not sufficient to induce LTF. The late expression of CREB1 is required and presumably requires one or more of the early gene products for the induction of additional genes necessary to maintain LTF. After $18 \mathrm{~h}$, the increased expression of CREB1 is not required for LTF observed at 24 or $48 \mathrm{~h}$, presumably because the products of CREB1-dependent gene expression have led to the enhancement of synaptic strength. Although this late $18 \mathrm{~h}$ increase is not necessary for LTF at 24 or $48 \mathrm{~h}$ (Fig. 5), it may be important for the protein synthesis-dependent maintenance of synaptic plasticity observed at longer times, such as $72 \mathrm{~h}$ after treatment (Casadio et al., 1999; Miniaci et al., 2008).

\section{5-HT-induced expression of CREB1 is required for long-term changes in excitability in sensory neurons}

CREB plays an important role in the conversion of short-term to long-term memory and the underlying short-term to long-term synaptic plasticity in both vertebrates and invertebrates (Alberini, 2009; Kandel, 2009). In Aplysia, long-term synaptic facilitation is also accompanied by a corresponding long-term change in excitability of SNs (Cleary et al., 1998), and this excitability can be mimicked by treatment of isolated SNs with 5-HT (Dale et al., 1987). CREB1 siRNA blocked LTE when measured $24 \mathrm{~h}$ later. This is the first evidence that CREB1 is required for long-term changes in excitability in Aplysia SNs. The finding that CREB1 mediates changes in cell excitability induced by $5-\mathrm{HT}$ is consistent with several lines of evidence from studies in vertebrates examining the relationship between CREB and excitability (Dong et al., 2006; Han et al., 2006; Jancic et al., 2009; Zhou et al., 2009). Increasing evidence suggests that changes in intrinsic excitability are a crucial component in learning and memory (Disterhoft and Oh, 2006; Benito and Barco, 2010; Mozzachiodi and Byrne, 2010).

Because LTE was observed in isolated SNs, this long-term change was intrinsic and did not depend on other circuit elements. Moreover, LTE was blocked when CREB1 siRNA was injected immediately after treatment. These data suggest that 5-HT-induced CREB1 synthesis is required for consolidation of both LTF and LTE. It is noteworthy that 5-HT-enhanced LTE lasts $<48 \mathrm{~h}$, whereas 5 -HT-induced LTF persists over $48 \mathrm{~h}$. This result suggests that the target proteins affecting these two processes diverge at some point downstream from CREB1. These findings are consistent with previous work demonstrating that 1 $\mathrm{d}$ training protocols that induce long-term sensitization in Aplysia produce an increase in excitability (Cleary et al., 1998), whereas $4 \mathrm{~d}$ training protocols do not (Wainwright et al., 2004).

In conclusion, the increased expression of CREB1 is required for both the consolidation phase of LTF and for LTE in presynaptic neurons. In addition, this CREB1 synthesis is required only during a limited time window that lasts $\sim 16-18 \mathrm{~h}$ after the induction of LTF. A large body of evidence from both vertebrates and invertebrates indicates that CREB is an important switch to convert short-term into long-term plasticity and memory. However, little attention has been paid to the ways in which the expression of CREB is regulated. The present results indicate that learning-induced regulation of CREB could have a powerful effect on the consolidation of long-term memory. In general, positive feedback loops are emerging as likely mechanisms to support LTM in a variety of systems (Atkins et al., 2004; Pastalkova et al., 2006; Tanaka and Augustine, 2008; Zhang et al., 2010). Therefore, understanding the dynamical properties of molecular positive feedback loops may provide 
insights into memory processes, such as the temporal properties of consolidation.

\section{References}

Alberini CM (2009) Transcription factors in long-term memory and synaptic plasticity. Physiol Rev 89:121-145.

Alberini CM, Ghirardi M, Metz R, Kandel ER (1994) C/EBP is an immediate-early gene required for the consolidation of long-term facilitation in Aplysia. Cell 76:1099-1114.

Angers A, Fioravante D, Chin J, Cleary LJ, Bean AJ, Byrne JH (2002) Serotonin stimulates phosphorylation of Aplysia synapsin and alters its subcellular distribution in sensory neurons. J Neurosci 22:5412-5422.

Artinian J, McGauran AM, De Jaeger X, Mouledous L, Frances B, Roullet P (2008) Protein degradation, as with protein synthesis, is required during not only long-term spatial memory consolidation but also reconsolidation. Eur J Neurosci 27:3009-3019.

Atkins CM, Nozaki N, Shigeri Y, Soderling TR (2004) Cytoplasmic polyadenylation element binding protein-dependent protein synthesis is regulated by calcium/calmodulin-dependent protein kinase II. J Neurosci 24:5193-5201.

Bartsch D, Casadio A, Karl KA, Serodio P, Kandel ER (1998) CREB1 encodes a nuclear activator, a repressor, and a cytoplasmic modulator that form a regulatory unit critical for long-term facilitation. Cell 95:211-223.

Bekinschtein P, Cammarota M, Igaz LM, Bevilaqua LR, Izquierdo I, Medina JH (2007) Persistence of long-term memory storage requires a late protein synthesis- and BDNF-dependent phase in the hippocampus. Neuron 53:261-277.

Benito E, Barco A (2010) CREB's control of intrinsic and synaptic plasticity: implications for CREB-dependent memory models. Trends Neurosci $33: 230-240$

Casadio A, Martin KC, Giustetto M, Zhu H, Chen M, Bartsch D, Bailey CH, Kandel ER (1999) A transient, neuron-wide form of CREB-mediated long-term facilitation can be stabilized at specific synapses by local protein synthesis. Cell 99:221-237.

Chin J, Angers A, Cleary LJ, Eskin A, Byrne JH (2002) Transforming growth factor betal alters synapsin distribution and modulates synaptic depression in Aplysia. J Neurosci 22:RC220(1-6).

Cleary LJ, Lee WL, Byrne JH (1998) Cellular correlates of long-term sensitization in Aplysia. J Neurosci 18:5988-5998.

Dale N, Kandel ER, Schacher S (1987) Serotonin produces long-term changes in the excitability of Aplysia sensory neurons in culture that depend on new protein synthesis. J Neurosci 7:2232-2238.

Dash PK, Hochner B, Kandel ER (1990) Injection of the cAMP-responsive element into the nucleus of Aplysia sensory neurons blocks long-term facilitation. Nature 345:718-721.

Davis HP, Squire LR (1984) Protein synthesis and memory: a review. Psychol Bull 96:518-559.

Disterhoft JF, Oh MM (2006) Learning, aging and intrinsic neuronal plasticity. Trends Neurosci 29:587-599.

Dong Y, Green T, Saal D, Marie H, Neve R, Nestler EJ, Malenka RC (2006) CREB modulates excitability of nucleus accumbens neurons. Nat Neurosci 9:475-477.

Emptage NJ, Carew TJ (1993) Long-term synaptic facilitation in the absence of short-term facilitation in Aplysia neurons. Science 262:253-256.

Fioravante D, Antzoulatos EG, Bryne JH (2008) Sensitization and habituation: invertebrate. In: Learning and memory: a comprehensive reference (Byrne JH, ed), Vol 4, pp31-45. Oxford: Elsevier.

Han MH, Bolaños CA, Green TA, Olson VG, Neve RL, Liu RJ, Aghajanian GK, Nestler EJ (2006) Role of cAMP response element-binding protein in the rat locus ceruleus: regulation of neuronal activity and opiate withdrawal behaviors. J Neurosci 26:4624-4629.

Hegde AN, Goldberg AL, Schwartz JH (1993) Regulatory subunits of cAMP-dependent protein kinases are degraded after conjugation to ubiquitin: a molecular mechanism underlying long-term synaptic plasticity. Proc Natl Acad Sci U S A 90:7436-7440.

Jancic D, Lopez de Armentia M, Valor LM, Olivares R, Barco A (2009) In- hibition of cAMP response element-binding protein reduces neuronal excitability and plasticity, and triggers neurodegeneration. Cereb Cortex 19:2535-2547.

Kandel ER (2009) The biology of memory: a forty-year perspective. J Neurosci 29:12748-12756.

Lee SH, Lim CS, Park H, Lee JA, Han JH, Kim H, Cheang YH, Lee SH, Lee YS, Ko HG, Jang DH, Kim H, Miniaci MC, Bartsch D, Kim E, Bailey CH, Kandel ER, Kaang BK (2007) Nuclear translocation of CAM-associated protein activates transcription for long-term facilitation in Aplysia. Cell 129:801-812.

Liu RY, Fioravante D, Shah S, Byrne JH (2008) cAMP response elementbinding protein 1 feedback loop is necessary for consolidation of longterm synaptic facilitation in Aplysia. J Neurosci 28:1970-1976.

Lonze BE, Ginty DD (2002) Function and regulation of CREB family transcription factors in the nervous system. Neuron 35:605-623.

Milhavet O, Gary DS, Mattson MP (2003) RNA interference in biology and medicine. Pharmacol Rev 55:629-648.

Miniaci MC, Kim JH, Puthanveettil SV, Si K, Zhu H, Kandel ER, Bailey CH (2008) Sustained CPEB-dependent local protein synthesis is required to stabilize synaptic growth for persistence of long-term facilitation in Aplysia. Neuron 59:1024-1036.

Mohamed HA, Yao W, Fioravante D, Smolen PD, Byrne JH (2005) cAMPresponse elements in Aplysia creb1, creb2, and Ap-uch promoters: implications for feedback loops modulating long term memory. J Biol Chem 280:27035-27043.

Montarolo PG, Goelet P, Castellucci VF, Morgan J, Kandel ER, Schacher S (1986) A critical period for macromolecular synthesis in long-term heterosynaptic facilitation in Aplysia. Science 234:1249-1254.

Mozzachiodi R, Byrne JH (2010) More than synaptic plasticity: role of nonsynaptic plasticity in learning and memory. Trends Neurosci 33:17-26.

Pastalkova E, Serrano P, Pinkhasova D, Wallace E, Fenton AA, Sacktor TC (2006) Storage of spatial information by the maintenance mechanism of LTP. Science 313:1141-1144.

Schacher S, Proshansky E (1983) Neurite regeneration by Aplysia neurons in dissociated cell culture: modulation by Aplysia hemolymph and the presence of the initial axonal segment. J Neurosci 3:2403-2413.

Sharma SK, Carew TJ (2004) The roles of MAPK cascades in synaptic plasticity and memory in Aplysia: facilitatory effects and inhibitory constraints. Learn Mem 11:373-378.

Sharp PA (2001) RNA interference-2001. Genes Dev 15:485-490.

Tanaka K, Augustine GJ (2008) A positive feedback signal transduction loop determines timing of cerebellar long-term depression. Neuron 59:608-620.

Upadhya SC, Smith TK, Hegde AN (2004) Ubiquitin-proteasome-mediated CREB repressor degradation during induction of long-term facilitation. J Neurochem 91:210-219.

Wainwright ML, Byrne JH, Cleary LJ (2004) Dissociation of morphological and physiological changes associated with long-term memory in Aplysia. J Neurophysiol 92:2628-2632.

Yamamoto N, Hegde AN, Chain DG, Schwartz JH (1999) Activation and degradation of the transcription factor C/EBP during long-term facilitation in Aplysia. J Neurochem 73:2415-2423.

Zhang F, Endo S, Cleary LJ, Eskin A, Byrne JH (1997) Role of transforming growth factor-beta in long-term synaptic facilitation in Aplysia. Science 275:1318-1320

Zhang Y, Smolen P, Baxter DA, Byrne JH (2010) The sensitivity of memory consolidation and reconsolidation to inhibitors of protein synthesis and kinases: computational analysis. Learn Mem 17:428-439.

Zhao Y, Hegde AN, Martin KC (2003) The ubiquitin proteasome system functions as an inhibitory constraint on synaptic strengthening. Curr Biol 13:887-898.

Zhou Y, Won J, Karlsson MG, Zhou M, Rogerson T, Balaji J, Neve R, Poirazi P, Silva AJ (2009) CREB regulates excitability and the allocation of memory to subsets of neurons in the amygdala. Nat Neurosci 12:14381443. 